\title{
Emprego de Subprodutos da Produção de Piches de Petróleo na Síntese de Nanoesferas de Carbono
}

\author{
Alexandre T. de Castro, Luiz D. de Castro \\ Centro Tecnológico do Exército, Núcleo de Competência para o Desenvolvimento de Tecnologia de Carbono \\ Cristina T. Andrade \\ Instituto de Macromoléculas, Universidade Federal do Rio de Janeiro
}

Resumo: Nanoesferas de carbono foram produzidas a partir de resíduos de petróleo, obtidos da produção de piches pela destilação de óleos decantados, pela técnica de deposição química de vapor a $1200{ }^{\circ} \mathrm{C}$, sem catalisadores, em um processo contínuo. Três resíduos foram selecionados de forma a representar a faixa de propriedades tipicamente encontradas para este material. Nitrogênio e argônio foram empregados como gases de arraste. As nanoesferas obtidas apresentaram propriedades semelhantes para os três resíduos diferentes, sendo a influência do precursor menos significativa que a do gás de arraste empregado. Os rendimentos obtidos, na faixa de 40-50\%, e a baixa sensibilidade do processo à composição do precursor indicam que o processo é adequado à utilização industrial.

Palavras-chave: Nanoesferas de carbono, resíduos de petróleo, deposição química de vapor.

\section{Use of Byproducts of Petroleum Pitch Production as Precursors for Synthesis of Carbon Nanospheres}

\begin{abstract}
Carbon nanospheres were produced from petroleum residues obtained in the production of petroleum pitches by distillation of decant oils, using chemical vapor deposition at $1200{ }^{\circ} \mathrm{C}$, without catalysts and in a continuous process. Three residues were selected to represent the typical variability in the properties of this material. Nitrogen and argon were used as carrier gases. The three residues yielded nanospheres of similar properties, which were more influenced by the nature of the carrier gas. The mass yields of $40-50 \%$ and the low dependence on precursor composition indicate that the process is suitable for large scale industrial use.
\end{abstract}

Keywords: Carbon nanospheres, petroleum residues, chemical vapor deposition.

\section{Introdução}

Nanoesferas de carbono apresentam estruturas concêntricas com diâmetros típicos entre $50 \mathrm{~nm}$ e $1 \mu \mathrm{m}$, constituindo uma forma intermediária entre os fulerenos e seus derivados, com diâmetros na faixa de 2 a $20 \mathrm{~nm}$, e as microesferas de carbono, com diâmetros acima de $1 \mu \mathrm{m}^{[1]}$. Apesar da maior parte dos estudos na área de nanomateriais de carbono serem direcionados para nanofilamentos (nanotubos e nanofibras), várias aplicações foram identificadas para as nanoesferas, incluindo suportes para catalisadores ${ }^{[2]}$ ou biosensores ${ }^{[3]}$, moldes para síntese de biomateriais ${ }^{[4]}$, e eletrodos para baterias de íon-lítio ${ }^{[5]}$. Assim como outros materiais de carbono, as nanoesferas apresentam potencial também para emprego em compósitos polímericos. Estudos iniciais revelaram sua possível aplicação em compósitos piezoresistivos à base de resinas epóxi, utilizados como sensores de pressão, e compósitos condutores à base de poliuretanos ${ }^{[6]}$.
A deposição química de vapor, empregando hidrocarbonetos puros como precursores, é o método mais difundido de síntese de nanomateriais de carbono, devido à sua simplicidade e facilidade de aumento de escala. Dois desafios ainda a superar, entretanto, são o alto custo destes materiais e o controle limitado das estruturas produzidas. Neste trabalho, é demonstrado que nanoesferas de carbono podem ser produzidas com alto rendimento a partir de resíduos da produção de piches de petróleo, sendo as propriedades do produto pouco afetadas pela composição do resíduo.

\section{Experimental}

Piches de petróleo foram obtidos pela destilação de óleos decantados (resíduos do craqueamento catalítico do petróleo) ${ }^{[7]}$ e a fração leve (condensado) recolhida. Três amostras de condensado foram selecionadas de forma a

Autor para correspondência: Alexandre T. de Castro, Centro Tecnológico do Exército, Núcleo de Competência para o Desenvolvimento de Tecnologia de Carbono, Av. das Américas, CEP: 23020-470, Guaratiba, Rio de Janeiro, RJ, Brasil. E-mail: tascheto@centroin.com.br 
representar a faixa de propriedades tipicamente encontradas para este material, sendo caracterizadas por picnometria, espectroscopia de infravermelho e ressonância magnética nuclear $^{[8]}$, extrografia ${ }^{[9]} \mathrm{e}$ análise elementar. Os condensados foram vaporizados e alimentados em um forno tubular vertical mantido a $1200{ }^{\circ} \mathrm{C}$, a uma taxa de $15 \mathrm{~mL} / \mathrm{h}$. Nitrogênio e argônio foram empregados como gás de arraste, a uma vazão de $1,3 \mathrm{~L} / \mathrm{min}$. As nanoesferas obtidas foram caracterizadas por termogravimetria em atmosfera oxidante, em fluxo de $20 \mathrm{~mL} / \mathrm{min}$ de ar e rampa de aquecimento de $10{ }^{\circ} \mathrm{C} / \mathrm{min}$, e microscopia eletrônica de varredura.

\section{Resultados e Discussões}

As propriedades dos condensados empregados como precursores encontram-se na Tabela 1. Espectros de FTIR e NMR condensados são qualitativamente idênticos, indicando que suas diferentes propriedades são resultantes das diferentes proporções dos hidrocarbonetos presentes em sua composição. Esta diferença é demonstrada pelos resultados de extrografia.

As nanoesferas apresentaram uma distribuição de diâmetros lognormal (Figura 1a, b), típica de processos de crescimento de partículas onde a velocidade de crescimento é proporcional a sua área superficial e sujeitos a fenômenos simultâneos de arraste e difusão ${ }^{[10]}$.

Os rendimentos obtidos foram aproxidamente $40 \% \mathrm{em}$ nitrogênio e $45 \%$ em argônio, com uma pequena variação para cada resíduo (Figura 1c). Para o resíduo A, a diferença entre o rendimento do processo com os dois gases de arraste foi mais pronunciada. A influência do gás de arraste no processo foi observada em outros estudos de produção de nanoesferas ${ }^{[11]}$ e nanotubos ${ }^{[12]}$, mas com respeito às propriedades dos produtos. Naqueles estudos o rendimento

Tabela 1. Resultados de picnometria, NMR e análise elementar e extrografia para os resíduos empregados ( $\mathrm{ND}=$ não detectado).

\begin{tabular}{lccc}
\hline & A & B & C \\
\hline Massa específica $\left(\mathrm{g} / \mathrm{cm}^{3}\right)$ & 1,055 & 1,022 & 0,954 \\
Aromaticidade NMR ${ }^{13} \mathrm{C}(\%)$ & 72,9 & 59,0 & 45,4 \\
Aromaticidade NMR ${ }^{1} \mathrm{H}(\%)$ & 34,4 & 25,0 & 15,3 \\
$\% \mathrm{C}$ (massa) & 87,17 & 87,70 & 84,72 \\
$\% \mathrm{H}$ (massa) & 7,53 & 8,55 & 9,71 \\
\% N (massa) & 0,38 & 0,67 & 0,19 \\
\% S (massa) & 0,81 & 0,66 & $\mathrm{ND}$ \\
\% O (por diferença) & 4,11 & 2,42 & 5,38 \\
$\mathrm{C} / \mathrm{H}$ & 0,972 & 0,861 & 0,732 \\
Frações determinadas por extrografia & & \\
$\quad$ Compostos alifáticos (\%) & 11,1 & 24,0 & 37,3 \\
Compostos aromáticos (\%) & 72,8 & 62,6 & 46,1 \\
Compostos heteroaro- & 6,0 & 7,4 & 2,8 \\
$\quad$ máticos nitrogenados (\%) & & & \\
Compostos heteroaro- & 2,1 & 2,4 & 3,3 \\
$\quad$ máticos sulfurados e & & & \\
polares (\%) & & & \\
\hline
\end{tabular}

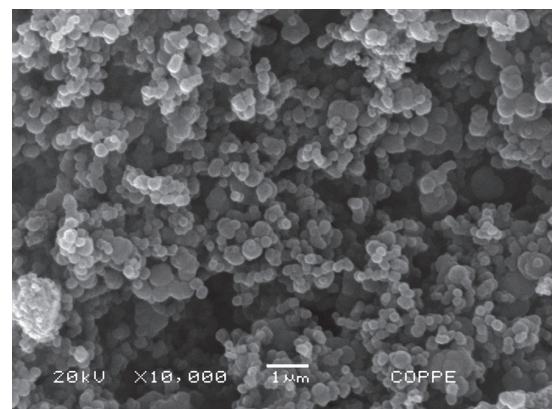

(a)

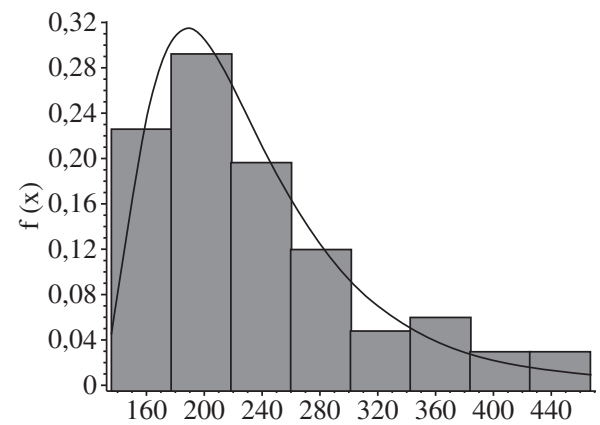

(b)

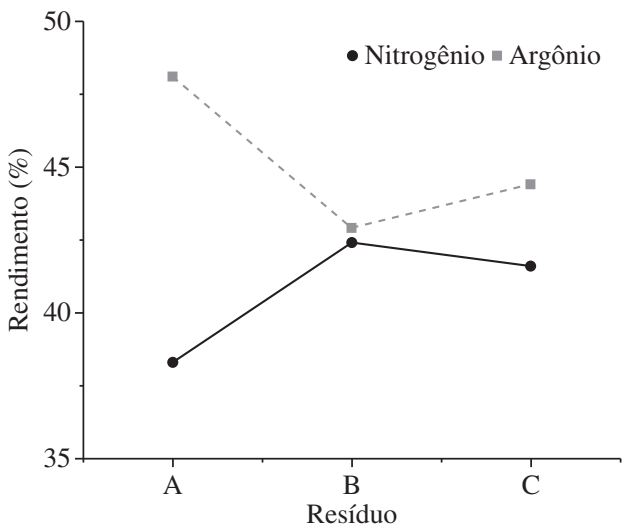

(c)

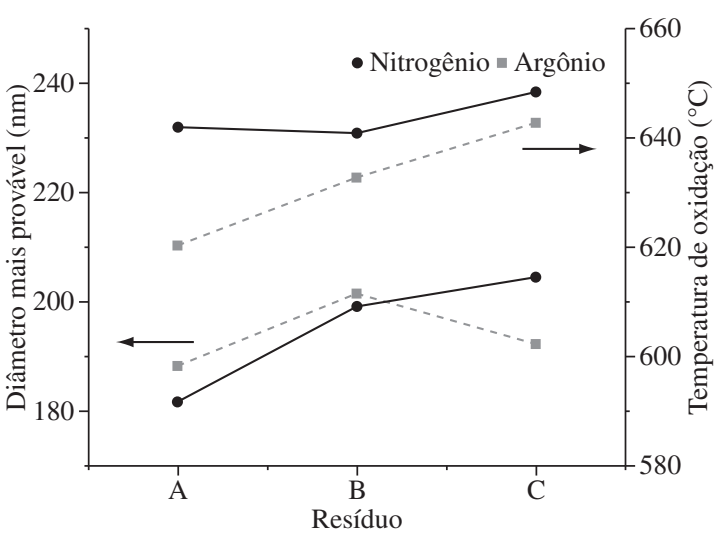

(d)

Figura 1. a) Microfotografia de nanoesferas obtidas em argônio a partir do resíduo A; b) Distribuição de diâmetros da mesma amostra (histograma e função densidade de probabilidade calculada considerando uma distribuição lognormal); c) Efeito do precursor e gás de arraste sobre o rendimento do processo; e d) Efeito do precursor sobre a temperatura de oxidação e diâmetro mais provável das nanoesferas. 
do processo não foi relatado. Apesar de inertes, as diferentes propriedades físicas dos gases afetam significativamente os processos de transferência de massa, calor e quantidade de movimento dentro da zona de reação ${ }^{[12]}$.

No caso deste estudo, a estabilidade das esferas à oxidação também foi claramente influenciada pelo gás de arraste, com esferas produzidas em nitrogênio apresentando o pico da derivada da curva de oxidação em temperaturas mais altas. A influência do precursor também dependeu do gás de arraste, com esferas produzidas em argônio apresentando maior variação entre os três resíduos (Figura 1c). Já os diâmetros apresentaram uma variação mínima, tanto para os dois gases de arraste como para os diferentes resíduos empregados como precursores, tendo seu valor mais provável na faixa de 180-200 nm em todos os casos (Figura 1d).

\section{Conclusões}

O uso de resíduos de petróleo de composição complexa como precursores para a produção de nanoesferas de carbono pela técnica de deposição química de vapor é tecnicamente viável. O processo de síntese é pouco dependente do resíduo utilizado, tendo sido observados rendimentos e propriedades semelhantes, para esferas de três resíduos de propriedades significativamente diferentes, sendo a influência do gás inerte de arraste maior que a do precursor. A pequena sensibilidade à composição do resíduo de petróleo usado como precursor, associada ao fato de os resíduos empregados serem obtidos como subprodutos da produção de piches de petróleo, significa que o processo tem grande potencial para a síntese de nanomateriais a um custo reduzido e em escala industrial.

\section{Agradecimento}

Os autores agradecem à Petrobras pelo apoio financeiro e fornecimento das amostras de óleo decantado.

\section{Referências Bibliográficas}

1. Serp, P.; Feurer, R.; Kalck, P.; Kihn, Y.; Faria, J. L. \& Figueiredo, J. L. - Carbon, 39, p.621 (2001).

2. Serp, P.; Feurer, F.; Kihn, Y.; Kalck, P.; Faria, J. L. \& Figueiredo, J. L. - J Mat Chem, 11, p.1980 (2001).

3. Chen X.; Zhang, J. J.; Xuan, J. \& Zhu, J. J. - Nano Res, 2, p.210 (2009).

4. Jagadeesan, D.; Deepak, C.; Siva, K.; Inandar, M.S. \& Eswaramoorthy, M. - J Phys Chem C, 112, p.7379 (2008).

5. Yoshizawa, N.; Tanaike, O.; Hatori, H.; Yoshikawa, K.; Kondo, A. \& Abe, T. - Carbon, 44, p.2558 (2006).

6. Shanov, V.; Schulz, M. \& Yeo-Heung, Y. - "Developing A New Grade of CTIC Carbon Nanosphere Chains and Processing This Material In Nanocomposites". Disponível em: <http://www.cleantechnano.com/pdf/ Developing_New_Grade_of_CleanTech_CNSC_ Nanocomposites_Edited\%20Version.pdf>

7. Dutra, C. H. M. C.; Castro, L. D. \& Andrade, C. T. Polim Ciência e Tec, 18, p.307 (2008).

8. Castro, A. T. - J Braz Chem Soc, 17, p. 1181 (2006).

9. Pereira, M. H. G. - "Análise cromatográfica de óleos pesados de petróleo", Dissertação de mestrado, Universidade Federal do Rio de Janeiro, Brasil (2008).

10. Kiss, L. B.; Söderlund, J.; Niklasson, G. A. \& Granqvist, C. G. - Nanotechnology, 10, p.25 (1999).

11. Qian, H.; Han, F.; Zhang, B.; Guo, Y.; Yue, J. \& Peng, B. - Carbon, 42, p.761 (2004).

12. Jost, O.; Gorbunov, A. A.; Möller, J.; Pompe, W.; Liu, X.; Georgi, P.; Dunsch, L.; Golden, M. S. \& Fink, J. Journal of Physical Chemistry B, 106, p.2875 (2002).

Enviado: 25/08/09

Reenviado: 02/10/09

Aceito: $11 / 10 / 09$ 